Original Research Paper

\title{
Preliminary on Human Driver Behavior: A Review
}

\author{
Xiao Yan ${ }^{1}$, Ashardi Abas ${ }^{1}$ \\ ${ }^{1}$ Universiti Pendidikan Sultan Idris, Malaysia.
}

Article History

Received:

04.10 .2020

Revised:

19.10.2020

Accepted:

24.11.2020

*Corresponding Author:

Ashardi Abas

Email:

ashardi@fskik.upsi.edu.com

This is an open access article, licensed under: $\mathrm{CC}-\mathrm{BY}-\mathrm{SA}$

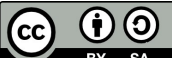

Abstract: Drowsiness is one of the main factors causing traffic accidents. Research on drowsiness can effectively reduce the traffic accident rate. According to the existing literature, this paper divides the current measurement techniques into subjective and objective ones. Among them, invasive detection and non-invasive detection based on vehicles or drivers are the main objective detection methods.Then, this paper studies the characteristics of drowsiness, and analyzes the advantages and disadvantages of each detection method in practical application. Finally, the development of detection technology is prospected, and provides ideas for the follow-up development of fatigue driving detection technology.

Keywords: Drowsiness, Fatigue Driving, Non-Visual, Visual. 


\section{Introduction}

Road traffic injuries are fatal disasters. According to the World Health Organization, 1.2 million people die on the road every year, and between 20 - 50 million sustain non-fatal injuries [1]. Among them, accidents caused by fatigue driving account for a considerable proportion of traffic accidents. The International Transport Forum at the OECD estimates that $20-30 \%$ of fatalities are due to driver sleepiness and fatigue [2], and in Europe, a survey across nineteen countries showed that the prevalence of falling asleep while driving in the previous 2 years is $17 \%$, and among those who fell asleep, the prevalence of sleep-related crashes was 7\% [3].

Drowsiness has always been a difficult point in road traffic safety management. The reason is that drowsiness is hidden, which is not easy to be detected as drunk driving. However, fatigue can also damage the driver's judgment, performance and reaction time like alcohol. Therefore, it is especially important to find suitable and accurate methods to quickly detect the drowsiness of drivers.

\section{Characteristics of Drowsiness}

There are two kinds of fatigue, physical fatigue, and mental fatigue. The former is mainly caused by the muscle can not rest, especially for manual workers. The latter is mainly due to busy work, mental tension which leads to inattention, distraction, lack of agility and unresponsiveness.

Driving fatigue means that long-term continuous driving causes the driver to feel sleepy and tired, resulting in physiological and psychological dysfunction. The main features are drowsiness, fatigue, reduced motivation, and unwillingness to continue driving, which may lead to a crash [3].

Physiological characteristics of drowsiness [4]:

- Visual system: drooping eyelids, blurred vision, decreased visual sensitivity, astringent eyes, sunken eyes, dark eyes, dull eyes, eyeball fibrillation and increased blinking times;

- Auditory system: unclear discrimination of the direction and volume of sound, hearing loss;

- Respiratory system: asthma, chest tightness, dry respiratory tract, and throat;

- Internal circulatory system: the heart rate slows down and blood pressure changes;

- Facial expression: inactive, stiff facial expression, muscle relaxation;

- Musculoskeletal system: soreness of waist, back pain, shoulder pain, muscle pain, joint pain, swollen hands, and feet;

- Central nervous system: decreased levels of mental activity;

- When the driver is fatigued, there will be a phenomenon of drowsiness, prolonged eye closure, dizziness, and even dozing.

Psychological characteristics of drowsiness [5]:

- The decrease in perception level: When the driver drives for 2 to 4 hours, the central nervous system will feel fatigued, and gradually the level of dynamic vision will decrease, the hearing will be slow, the illusion will occur, and even hallucinations will occur. Under the fatigue driving state, the driver recognizes that the traffic sign is $30 \%$ more than the normal state, and the ability is greatly reduced.

- Weak focus consciousness: the focused consciousness related to driving behavior will be weakened due to fatigue, but other related consciousness activities will be enhanced, pay attention to dispersion, pay attention to stability decline, and receive external information slowly and scorn; the scope of attention is narrowed; attention to the distribution and transfer of difficulties, misreading, missing information to see more information; often lose important traffic information, while paying attention to secondary traffic information, visual and auditory attention to processing capacity, involuntary attention to decline.

- Memory and thinking mistakes: In the case of excessive fatigue, it is detrimental to the driver's thinking and memory, the judgment of the external things is declining, the mind is not clear, and the operating procedures are often forgotten, and the traffic rules are violated. The situation behind the car, forgetting to turn on the turn signal when turning, and taking the wrong route.

- Prolonged reaction time: before fatigue and before fatigue, the driver's reaction time increases by an average of 0.1 seconds, and some even more than 2 times, especially the response time of the red signal increases significantly. 
- Operation error: The driver's muscle contraction adjustment ability will be reduced due to long-term driving. Therefore, after driving fatigue, the coordination of driving operation is damaged, the accuracy of motion is reduced, the degree of automation is reduced, and the operation is weak. The operation is uncoordinated, the brakes and throttles are not stable, and the direction, shifting and other operations are not flexible.

- When fatigue occurs, the driver's judgment, driving, and operation errors are much higher than usual, for example, the judgment of traffic conditions and road conditions is wrong. To deal with potential hazard judgment errors or improper methods.

According to the physiological and psychological characteristics of fatigue driving, it can be detected and judged in a targeted manner and used as the basis for analysis and discrimination of driving fatigue.

\section{Detection Methods}

At present, the developed driving fatigue detection technology can be roughly classified into subjective detection and objective detection according to different properties. Subjective testing is mainly carried out by the driver according to the self-reporting of the fatigue scale or the method of scoring by others; the objective detection mainly assesses the degree of fatigue by detecting the physiological and behavioral indicators of the driver during driving.

\subsection{Subjective Detection}

Subjective self-assessment mainly relies on questionnaires or self-reported forms of fatigue symptoms to get the driver's fatigue level. The Pearson fatigue scale is a commonly used subjective fatigue questionnaire. From the time it is about to fall down to the level of energy, it is divided into levels. The subjects choose the closest level according to their own situation as the mental state. Subjective assessment is to observe the external performance of the subject, such as whether to yawn and frequency, etc., to estimate the degree of fatigue.

Regardless of whether it is self-evaluation or his evaluation, subjective evaluation has subjective scoring, difficulty in unifying the scoring standards and being affected by various personal abilities such as memory. There are even many shortcomings such as the driver deliberately concealing the true feelings and guessing the meaning of the questions. However, due to the simple subjective evaluation operation, in many cases, fatigue can be detected as an auxiliary means.

\subsection{Objective Detection}

Objective detection is to obtain indicators in the driving process with certain auxiliary tools, and these indicators are objective. The ordinary index detection method may be broadly divided into two categories: non-invasive (non-contact) and invasive (in direct contact with the driver). The main alertness signals are given in Table 1.

Table 1. The Main Alertness Signals

\begin{tabular}{|c|c|c|c|}
\hline Feature & Category & Alertness and Technique & Sensors \\
\hline $\begin{array}{c}\text { Non- } \\
\text { Intrusive }\end{array}$ & $\begin{array}{c}\text { Vehicle- } \\
\text { Based }\end{array}$ & $\begin{array}{l}\text { (1) Lane departure: } \\
\text { Based on the visual sensor, speed sensor, steering wheel rotation } \\
\text { angle sensor to monitor the driving route of the vehicle, analyze } \\
\text { and judge whether the driver has a mental state such as } \\
\text { distraction and sleepiness. The camera collects the identification } \\
\text { line of the driving lane in real time and analyzes the position of } \\
\text { the current car in the lane through image processing. If the car is } \\
\text { detected to deviate from the lane, the driver will be alerted in } \\
\text { some way to give the driver time to react. } \\
\text { (2) Steering wheel movement } \\
\text { When the driver is tired, the spirit is dispersed, and the steering } \\
\text { wheel pressure will become smaller. Statistical analysis of the } \\
\text { changes in pressure, the pressure to find an important point to } \\
\text { reflect changes in driver fatigue states. Based on the steering } \\
\text { wheel sensor, the steering wheel is rotated as the input signal, and }\end{array}$ & $\begin{array}{l}\text { Attached } \\
\text { to the } \\
\text { Vehicle }\end{array}$ \\
\hline
\end{tabular}




\begin{tabular}{|c|c|c|c|}
\hline & & $\begin{array}{l}\text { the chaotic theory is introduced to analyze the driver's mental } \\
\text { state. }\end{array}$ & \\
\hline & $\begin{array}{l}\text { Driver- } \\
\text { Based }\end{array}$ & $\begin{array}{l}\text { (1) Facial expression: } \\
\text { When fatigue occurs, the facial expression of the person is } \\
\text { reduced by the degree of eyelids, the upper eyelid is dropping, } \\
\text { the iris area is covered by the upper eyelid; the eyebrows are } \\
\text { lowered, the blinking is passive; the corner of the mouth is } \\
\text { drooping and contracting inward. Based on the visual sensor, the } \\
\text { driver's average eyelid closing frequency, the frequency of eye } \\
\text { gaze flicker, and the change in facial expression (the frequency of } \\
\text { yawning) are detected to determine whether it is fatigued. } \\
\text { Through the template matching, the curvature of the upper eyelid } \\
\text { of the eye and the projection of the closed eyes are calculated, the } \\
\text { state of the eye is detected, the state of the eye is recognized, and } \\
\text { the fatigue of the driver is detected. } \\
\text { (2) Head movement: } \\
\text { Measuring a change in the driver's seat over the sensor array } \\
\text { between the electrode of the capacitor, determining the position } \\
\text { of the driver's head in three-dimensional space, and the change of } \\
\text { the head position of each time period is determined fatigue. } \\
\text { (3) Surface Electromyography: } \\
\text { EMG Fourier frequency spectrum of the surface of driver fatigue } \\
\text { occurs to varying degrees to the left, the EMG power spectrum } \\
\text { value of the frequency shift to lower frequency, the low- } \\
\text { frequency signal energy is increased, the high-frequency signal } \\
\text { energy Relative reduction } \\
\text { (4) Mouth state: } \\
\text { When using machine vision to check the driver's behavior, it is a } \\
\text { key to determine the state of the mouth. Since the degree of } \\
\text { opening of the mouth in normal, talking and lethargy is different, } \\
\text { according to this fact, the classifier is used to extract the shape } \\
\text { and position of the mouth, then the geometrical characteristics of } \\
\text { the mouth area are used as feature values, and all these features } \\
\text { are composed. The feature vector is input into the three-level } \\
\text { network to obtain three different mental states. }\end{array}$ & \\
\hline Intrusive & & $\begin{array}{l}\text { (1) EEG: paste electrode through the head driver brainwave signal } \\
\text { acquisition, compared with the average power spectral density of } \\
\text { the brain waves of different frequency bands when the driver } \\
\text { awake drowsiness, the fatigue of the driver is determined } \\
\text { (2) ECG: When the driver is tired, there is a significant decrease in } \\
\text { heart rate. There are many analytical methods and evaluation } \\
\text { indicators for heart rate variability. The more common ones are } \\
\text { time domain analysis and frequency domain analysis. The time } \\
\text { domain method is to calculate statistically significant indicators } \\
\text { based on intervals and use these indicators to represent changes. } \\
\text { (3) EOG: The driver is affected by fatigue or lethargy, facial muscle } \\
\text { tension is reduced, eyelid movement is slow, and closed eyes or } \\
\text { microsleep time is longer. The electro-oculogram requires an } \\
\text { electrode, two of which are placed above and below the eye to } \\
\text { obtain data in the vertical direction, and the other two are placed } \\
\text { on the left and right sides of the eye to obtain horizontal data. The } \\
\text { use of EO signals can give more features of eyelid movement, } \\
\text { such as accurate eye-closing time, intensity and speed of each } \\
\text { blink, and more important eye movements, including slow eye } \\
\text { movements and rapid eye movements. Combining all the features } \\
\text { of eye electricity can make the alert estimate more stable and } \\
\text { accurate. }\end{array}$ & $\begin{array}{c}\text { Attached } \\
\text { to the } \\
\text { Driver }\end{array}$ \\
\hline
\end{tabular}




\section{Discussion of Effectiveness}

\subsection{Invasive Detection Effectiveness}

In the intrusive detection method, the measured values are obtained by adding electronic devices to the skin to access the condition of the driver. These include electroencephalogram (EEG), electrocardiogram (ECG) and electro-oculogram (EOG) [6] and the like.

EOG has some limitations, such as lighting conditions and sunglasses, which may affect the accuracy of the template matching technique. Another disadvantage of template matching techniques is that if the template is distorted due to image processing, it will fail. This technique is not enough to detect the driver's drowsiness with an accuracy rate of about $80 \%$.

The EEG is called the "gold standard" for measuring sleepiness. According to it, the driver's drowsiness can be easily judged. Most of the research results show that the $\delta$ and $\theta$ waves increase greatly when drowsiness, while the $\alpha$ wave and $\beta$ wave change little. Many studies have been detected using the EEG signal from postmen who carried out several tests on the vehicle simulator and the actual vehicle. It is effective to detect the drowsiness state of drivers [7]. In order to detect EEG signals, the drive must wear a micro-electrode helmet when driving, and this helmet can get data from the brain. The reliability and accuracy of detecting drowsiness of drivers with ECG and EOG were over $90 \%$ [8]. Although these devices produce highly accurate results, they are not widely accepted due to their practical limitations.

\subsection{Effectiveness of Non-Invasive Detection}

Among the non-invasive detection methods, one is the system analysis method based on vehicle control, including steering wheel movement, lane departure measurement, and the like. Vehicle-based methods are non-invasive, but because they are susceptible to driver driving skills, driving habits, speed, vehicle characteristics, and road conditions, they may not be as reliable in accurately detecting drowsiness [9].

The other type is based on behavioral measurement. Information is obtained by detecting a slight change in the driver's facial expression using a camera. Because behavioral measures are non-invasive in nature, they are becoming a popular method of detecting drowsiness. However, the detection method has certain limitations. For example, the analysis of the state of the eye, due to lighting conditions, sunglasses and other factors can affect the accuracy of the template matching technology. If the mode is distorted due to image processing, it will fail. Therefore, the correct rate of this method is about $80 \%$ [10]. The mouth state can be analyzed according to the face detection algorithm to analyze the yawn to determine the degree of drowsiness [11]. The yawning mouth is larger than the normal mouth, so the chance of fatigue driving is found to be higher. However, if the driver speaks or sings while driving, it is difficult to distinguish the two behaviors from yawning, as both of these conditions may cause the mouth to detect false positives. This is the main problem in detecting yawns in such systems. In a broad sense, many studies have detected yawns based on the change in mouth opening rate and mouth contour area. According to the survey, the overall accuracy of the technology is about $80 \%$ [12].

In general, using a single fatigue detection method, the indicators obtained are not comprehensive enough, and it is necessary to comprehensively test the indicators that can effectively detect driving fatigue from multiple angles. At present, the method of signal fusion and the image processing technology based on the neural network have been widely promoted in the fatigue detection method.

\section{Conclusion}

This paper reviews the characteristics, detection methods and effectiveness of fatigue driving. The invasive physiological signal detection method can reflect the fatigue state of the human body more accurately because of its objectivity. It has always been considered as a good indicator for detecting driving fatigue. However, it has to be attached to electrodes at different positions of the human body, which is not easy to be accepted by people.

A certain amount of equipment and technology is required; it is difficult to detect in real time due to limitations of the equipment. In view of the above problems, in order to further study driving fatigue through physiological signals, improvements in detection equipment can be performed, such as using physiological signals without electrodes to improve the real-time performance of the detection equipment. The improvement of non-invasive signal acquisition methods and the correlation between signal and fatigue are expected to greatly promote the advancement of physiological signal 
Xiao Yan, Ashardi Abas.

Preliminary on Human Driver Behavior: A Review.

International Journal of Artificial Intelligence, vol. 7, no. 2, pp. 29-34, December 2020. DOI: 10.36079/lamintang.ijai-0702.146

detection methods. With the deepening of research work, as the most effective detection method, the vehicle driving detection technology based on physiological signals will be further improved, and the promotion of practical testing equipment will be promoted.

\section{References}

[1] I. T. Forum, Road Safety Annual Report 2017, OECD iLibrary, 2017.

[2] M. Goncalves, R. Amici, T. Lucas, F. Kerstedt, J. Cirignotta, D. Horne, W. T. Lger, M. McNicholas, J. T-S. Partinen, P. Peigneux, and L. a. Grote, "Sleepiness at the wheel across Europe: a survey of 19 countries," Journal of Sleep Research, vol. 24, no. 3, 2001.

[3] P. A. Hancock, 3.1 Active and Passive Fatigue States [J]. Stress, workload, and fatigue.

[4] C. George, "Driving and automobile rashes in patients with obstructive sleep apnoea/hypopnoea syndrome [J]," Thorax, vol. 59, no. 9, 2004.

[5] S. Kaplan, A. G. Yavuz, and Y. Karalurt, "Driver Behavior Analysis for Safe Driving: A Survey," ITS, vol. 16, no. 6, 2015

[6] L. Lin, "Driver fatigue detection based on eye state, Technol," Health Care, vol. 23, no. 2, 2015.

[7] D. Costa, "A fadiga na condução," in Autoridade Nacional Segurança Rodoviária, 2014.

[8] Y. Saito, M. Itoh, and T. Inagaki, "Driver assitance system with a dual control scheme: effectiveness of identifying driver drowsiness and preventing lane departure accidents," IEEE Transactions on Human-Machine Systems, vol. 46, no. 5, 2016.

[9] M. Sabet, R. Zoroofi, K. Haghighi, and M. Sabbaghian, "A new system for driver drowsiness and distraction detection," in Iranian Conference on Electrical Engineering, Tehran, 2012.

[10] I. H. Choi, and Y. G. Kim, "Head pose and gaze dircetion tracking for detecting a drowsy driver," in International Conference on Big Data and Smart Computing, Bangkok, 2014.

[11] Z. Ma, B. C. Li, and Z. Yan, "Wearable driver drowsiness detection using electrooculography signal," in IEEE Topical Conference on Wireless Sensors and Sensor Networks, 2016.

[12] T. Hwang, M. Kim, S. Hong, and K. S. Park, "Driver drowsiness detection using the in-ear EEG (electroencephalogram)," in IEEE 38th Annual International Conference of the Engineering in Medicine and Biology Society, Orlando, 2016. 